\title{
Multi-carrier Index Modulation Method Based on PSWF-CPM Signal
}

\author{
Dawei Yang ${ }^{12+}$, Chuanhui Liu ${ }^{1}$ and Lei Zhang ${ }^{1}$ \\ ${ }^{1}$ Naval Aviation University, China \\ ${ }^{2}$ Unit 91439 of the PLA, China
}

\begin{abstract}
Index modulation is a hot spot in the research of multi-carrier modulation technology in wireless communication, on the basis of the traditional multi-carrier index modulation method in frequency domain, in this paper, the signal of prolate spheroidal wave functions (PSWF) with the best energy concentration in time-frequency domain is taken as the multi-carrier transmission waveform, continuous phase modulation (CPM) symbol mapping mode is adopted, a multi-carrier index modulation method based on PSWF-CPM signal is proposed. At the receiving end, the method of greedy detection (GD) and CPM difference decomposition mapping is used to realize the detection and demodulation of low complexity signals. The method can effectively reduce the peak-to-average power ratio (PAPR) of PSWF-CPM modulation signal according to the change of CPM modulation index, and the GD detection method is insensitive to imperfect channel state information (CSI), and the implementation complexity is relatively low.
\end{abstract}

Keywords: index modulation, PSWF, CPM, greedy detector.

\section{Introduction}

Index modulation (IM) is a hot technology in wireless communication research nowadays, which can make 5G wireless communication system have higher spectrum efficiency and energy efficiency [1]. Index modulation transmits information by selecting different index numbers, and these index resources can be physical or virtual. In recent years, scholars have been exploring in the field of multi-carrier index modulation, and have done a lot of research on orthogonal frequency division multiplexing with index modulation or so-called OFDM with Index Modulation (OFDM-IM). With frequency carrier as index resource, it has higher energy efficiency and spectrum efficiency than traditional typical OFDM technology [2]. Moreover, the number of active sub-carriers in each sub-carriers group can be configured to realize the on-demand adjustment between the bit error rate (BER) performance and the spectral efficiency of the system [3]. However, OFDM-IM technology still uses rectangular pulse as sub-carriers, and its disadvantages in out-of-band radiation and out-of-band attenuation still exist [4], and the maximum likelihood (ML) detection algorithm is relatively complex.

Prolate spheroidal wave functions (PSWF) was originally a set of non-sinusoidal functions proposed by D. Slepian and H. O. Pollak of Bell Labs, this function signal has excellent basic characteristics such as flexible time-bandwidth product controllability, optimal time-frequency energy aggregation [5], etc. It has been applied and researched in wireless communication, ultra-wideband communication (UWB), satellite communication [6-8], etc. Unlike the time bandwidth product of the rectangular window adopted by the OFDM signal is a constant, the time bandwidth product of the PSWF signal is flexible and variable, and can generate any time bandwidth product signal, and utilize PSWF of different orders as sub-carrier signal under that condition of fixed time bandwidth product, and its own best band-limited signal advantage, it can provide a new waveform design scheme for $5 \mathrm{G}$ mobile communication, satellite communication. CPM signal is a kind of digital angle modulation method, which has the characteristics of high power and spectrum efficiency, constant envelope and insensitivity to nonlinear characteristics [9-10]. In OFDM system, CPM symbol mapping can also reduce the peak-to-average power ratio (PAPR) of its modulated signal to some extent [11].

\footnotetext{
+ Corresponding author. Tel.: +86 18641167526.

E-mail address: yangdawei_2016@163.com.
} 
In this paper, combine that excellent basic characteristics of PSWF signal with the advantage of CPM modulation signal, a design scheme of index multi-carrier transmission waveform with PSWF signal, CPM is adopted as the index symbol mapping method, the multi-carrier index modulation method based on PSWFCPM is proposed, or so-called PSWF-CPM-IM. The low complexity PSWF-CPM-IM signal demodulation is realized by combining greedy detection (GD) with CPM differential detection, the power spectral density (PSD) characteristics, PAPR, BER performance and implementation complexity of PSWF-CPM-IM signal are simulated and analyzed.

\section{PSWF-CPM-IM Signal Modulation Method}

In this method, at the signal transmitting end, firstly, the number of PSWF sub-carriers signal paths $c-l, l \in[1, c-1]$ for information loading is generated (where: $c$ represents the time bandwidth product of baseband PSWF signal $c=B T(\mathrm{~Hz} \cdot \mathrm{s})$, and $T$ is the PSWF sub-carriers period). The number of signal packets $G$, the number of signal paths $N$ in each group, the number of active sub-carriers $K$, the PSWF signals with high energy concentration are optimized and grouped. Secondly, the index bit is used to select the active PSWF sub-carriers position, and use CPM to map symbol information. Then, the information mapped by CPM is loaded on $G$ groups of $N$-order PSWF sub-carriers, among them, the number of PSWF sub-carriers used for transmitting CPM symbol data is $K$, and the number of null sub-carriers is $(N-K)$. Finally, each group of PSWF sub-carriers loaded with CPM symbol mapping information is superimposed to generate PSWFCPM-IM modulation signals. The principle diagram of modulation signal generation is shown in Figure 1.

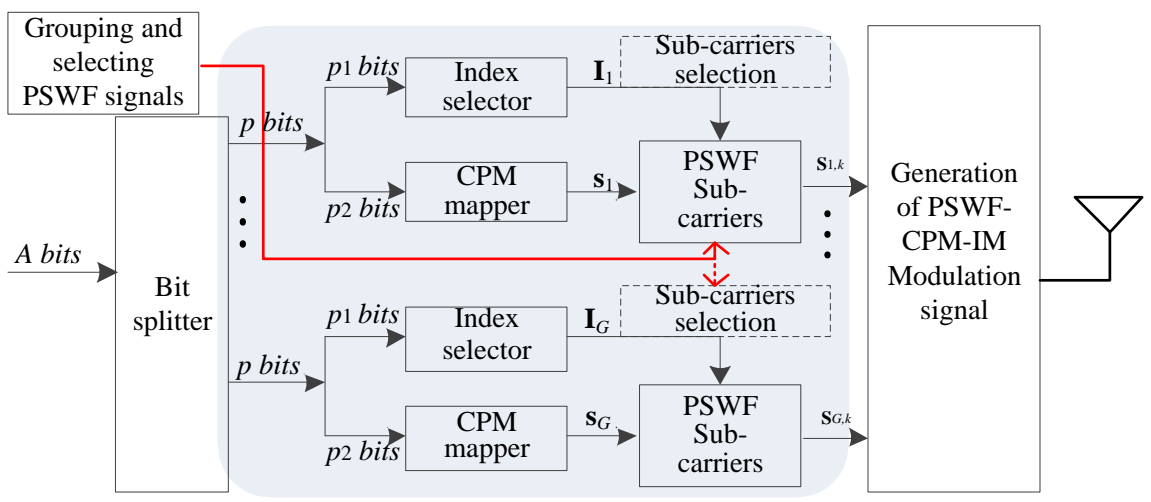

Fig. 1: Principle Diagram of PSWF-CPM-IM Signal Modulation

where, the generation of PSWF sub-carriers signal is obtained by the following PSWF integral definition formula:

$$
\int_{-T / 2}^{T / 2} \psi_{i}(c, \tau) \frac{\sin \Omega(t-\tau)}{\pi(t-\tau)} d \tau=\psi_{i}(c, \tau) \lambda_{i}(c)
$$

where $\psi_{i}(c, t)$ is a common $i$-order PAWF with band limited to $[-B, B]$ and concentrated distribution in time domain interval $[-T / 2, T / 2], i=0,1,2, \ldots, n-1, c=B T(\mathrm{~Hz} \cdot \mathrm{s})$ is its time bandwidth product, $\lambda_{i}$ is the eigenvalue corresponding to $\psi_{i}(c, t)$,For example, $\psi_{0}(c, t)$ is the 0 -order PSWF signal, $\lambda_{0}$ is the eigenvalue corresponding to $\psi_{0}(c, t)$, and PSWF signals of different orders correspond to their respective eigenvalues.

The generated $i$-order PSWF sub-carriers signals are taken as the first $c-l$-order PSWF signals, the $c-l, l \in[1, c-1]$ branch signals are equally divided into $G=\lfloor(c-l) / N\rfloor$ groups, and each group has $N$ PSWF sub-carriers signals. If each PSWF symbol has $M$ sub-carriers to transmit $A$-bits information, then, at first, the $A$-bits information is divided into $G$ groups after passing through a bit splitter, and each group has $p$-bits information, where $A=p G$. Each group of $p$-bits information is mapped to a PSWF sub-carriers group with length $N$, where $N=M / G$. The $p$-bits information transmitted to each group is divided into $p_{1}$ and $p_{2}$, where $p_{1}$ is an index bit, which is used to control the position of the active PSWF sub-carriers, here, look-up table (LUT) is used to realize the mapping between index bits and active sub-carriers positions. Establishing a table with the size of $\kappa=2^{p_{1}}$ at the sending end and the receiving end, at the sending end, the $p_{1}$ bit is mapped to the position of the sub-carriers by looking up the table, at the receiving end, according to the position of the active sub-carriers, the table is looked up to obtain the corresponding bit, Table 1 is the table 
size $\kappa=4$, when $(N, K)=(4,3)$, the PSWF sub-carriers index scheme corresponding to the look-up table method, where $K$ represents the number of active sub-carriers, $p_{2}$ is used to generate modulation symbols, in each group of sub-carriers, not every sub-carriers transmits constellation symbols, but there are $K$ transmission constellation symbols in $N$ sub-carriers. The remaining $(N-K)$ sub-carriers are null sub-carriers, the constellation mapping method here adopts CPM mapping with phase coding characteristics, and the constellation map is related to the CPM modulation index, and the CPM mapping constellation map and phase tree are shown in Figure 2 (taking the modulation index $h=0.5$ as an example).

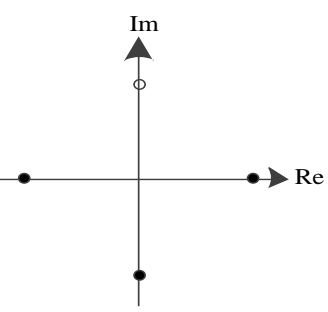

(a) CPM Mapping Constellation

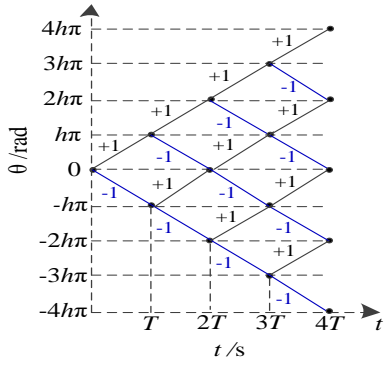

(b) CPM Mapping Phase Tress

Fig. 2: CPM Mapping Constellation Map and Phase Tree

Table 1: Transmitting mapping table of $\operatorname{IMLUT}(N, K)=(4,3)$

\begin{tabular}{|c|c|c|}
\hline Input bits & Active sub-carriers number & Mapping sub-carriers groups \\
\hline$\left[\begin{array}{ll}0 & 0\end{array}\right]$ & $\{1,2,3\}$ & {$\left[s_{g, 1} s_{g, 2} s_{g, 3} 0\right]^{T}$} \\
\hline$\left[\begin{array}{ll}0 & 1\end{array}\right]$ & $\{1,2,4\}$ & {$\left[s_{g, 1} s_{g, 2} 0 s_{g, 3}\right]^{T}$} \\
\hline$\left[\begin{array}{ll}1 & 0\end{array}\right]$ & $\{1,3,4\}$ & {$\left[s_{g, 1} 0 s_{g, 3} s_{g, 2}\right]^{T}$} \\
\hline$\left[\begin{array}{ll}1 & 1\end{array}\right]$ & $\{2,3,4\}$ & {$\left[0 s_{g, 1} s_{g, 2} s_{g, 3}\right]^{T}$} \\
\hline
\end{tabular}

\section{PSWF-CPM-IM Signal Detection and Demodulation Method}

At present, the frequency domain index modulation detection mainly includes ML detection and loglikelihood ratio (LLR) detection, in order to further reduce the detection complexity, this paper applies GD detection method to PSWF-CPM--IM signal detection. GD detection was originally applied to generalized OFDM multi-carrier index modulation by James Crawford and Youngwook Ko of Queen's University Belfast [12]. Here, it can be divided into two parts: the index position (bits) of the PSWF sub-carriers signal and the symbol bits of CPM mapping, the principle of PSWF-CPM-IM signal demodulation and detection method is shown in Figure 3, and the specific implementation process of detection is as follows:

Firstly, the modulated signal after passing the channel is correlated with PSWF sub-carriers signal, so that CPM symbol mapping information is unloaded from PSWF sub-carriers. It should be noted here that in frequency domain index modulation, PSWF signal is used as sub-carriers to load symbol mapping information, at the receiving end, only the correlation process with each group of orthogonal PSWF subcarriers signals is needed (this process will not affect the index modulation detection performance). That is, the detection performance of index modulation has nothing to do with the sub-carriers waveform loaded by symbol information. Therefore, for the detection of PSWF-CPM-IM signal, the data directly after the premodulation symbol data before PSWF sub-carriers loading is used as the detection statistics for analysis.

Assume that the modulated signal is superimposed by Rayleigh fading and Gaussian noise [13],where the channel matrix $\mathbf{H}=\operatorname{diag}\{h(1), \ldots, h(N)\}$ has its entries being complex Gaussian random variable with zero mean and unit variance, i.e., $h(\alpha) \sim C N(0,1), \alpha=1, \ldots N$, and $\boldsymbol{\eta}$ denotes the additive white Gaussian noise (AWGN) vector with $\eta(\alpha) \sim C N\left(0, N_{0}\right), \alpha=1, \ldots N$. Then the received signal $\mathbf{y}$ after passing the channel can be expressed as:

$$
\mathbf{y}=\mathbf{H} \mathbf{x}+\boldsymbol{\eta}
$$

To detect the position of the active PSWF sub-carriers of each group, at the receiving end, the noisy signals are divided into $G$ groups, and for the g group, the data are:

$$
\mathbf{y}_{g}=\left[y_{g}(1), y_{g}(2), \ldots, y_{g}(N)\right]^{T}
$$


where $\mathbf{y}_{g}=\left[y_{g}(1), y_{g}(2), \ldots, y_{g}(N)\right]^{T}$ is the data after $\mathbf{x}_{g}=\left[x_{g}(1), x_{g}(2), \ldots, x_{g}(N)\right]^{T}$ passes through the channel. After obtaining the detection statistics information $\left.\mathbf{y}_{g}, 1\right)$ Let a residual vector $\mathbf{z}_{0}=\mathbf{y}$, a demodulated vector $\mathbf{r}_{0}$ is set to a zero vector, i.e., $\mathbf{r}_{t}=\left[r_{t}(1), \ldots, r_{t}(N)\right]$ with $r_{0}(\alpha)=0, \forall \alpha$, and the iteration count $t=0$ where $t=1, \ldots, K .2)$ The sub-carriers with the greatest received power is estimated as one of the PSWF activated sub-carriers and its index $\hat{\alpha}$ is given by:

$$
\hat{\alpha}=\arg \max \left|z_{\alpha}(\alpha)\right|^{2}
$$

3) Let $r_{t}(\hat{\alpha})=z_{t}(\hat{\alpha})$ and $z_{t}(\hat{\alpha})=0$, and increment $t$ by $t=t+1$. 4) Repeat parts 2) and 3) until $t=K$. Then, the index bits are recovered by the look-up table method, use the look-up table method to recover the index bits $\mathrm{p}_{1}$ through the corresponding $\mathbf{r}_{K}$, with $N=4$ and $K=3$ as columns, the corresponding relationship between $\mathbf{r}_{K}$ and $p_{1}$ bits is shown in Table 2 .

Table 2: Receiving mapping table of IM LUT $(N, K)=(4,3)$

\begin{tabular}{|c|c|}
\hline Sub-carrier index $\mathbf{r}_{K}$ & $p_{1}$ bits \\
\hline 1110 & 00 \\
\hline 1101 & 01 \\
\hline 1011 & 10 \\
\hline 0111 & 11 \\
\hline
\end{tabular}

Assume that each non-zero symbol has the average transmit power of $\varphi E_{\mathrm{s}}$, where $\varphi=N / K$ is the power allocation coefficient and $E_{s}$ is the average power per sub-carrier. Thus, the average signal-to-noise ratio (SNR) per active sub-carrier is given by $\bar{\gamma}=\varphi E_{\mathrm{s}} / N_{0}$. Grouping $\mathbf{y}$ and removing power gain, on each PSWF sub-carrier, the CPM complex mapping information $\hat{\mathbf{x}}$ loaded on the active sub-carriers is separately estimated by maximum likelihood decision, which is expressed as:

$$
\hat{\mathbf{x}}(\hat{\alpha})=\arg \min _{x(\hat{\alpha}) \in S}|y(\hat{\alpha})-\hat{h}(\hat{\alpha}) x(\hat{\alpha})|^{2}, \forall \hat{\alpha}
$$

where $\alpha=1, \ldots N$, in particular, the channel estimate for each sub-carrier $\hat{h}(\alpha)$ is given by:

$$
\hat{h}(\alpha)=h(\alpha)-e(\alpha)
$$

where $e(\alpha) \sim C N\left(0, \varepsilon^{2}\right)$ denotes the channel estimation error, where $\varepsilon^{2}$ is the channel state information (CSI) error variance. Assume that $\hat{h}(\alpha)$ and $e(\alpha)$ are independent, which leads to $h(\alpha) \sim C N\left(0,1-\varepsilon^{2}\right)$. If $\varepsilon^{2}=0$, then it is considered to be an perfect CSI, $\varepsilon^{2}$ is a constant value, as a fixed CSI, $\varepsilon^{2}=1 /\left(\xi+10 \log \left(E_{\mathrm{s}} / N_{0}\right)\right)$, as variable CSI, where $\xi$ is the minimum mean square error (MMSE) channel estimator yields the error variance [13].

After parallel-to-serial conversion of the packet complex mapping information $\hat{\mathbf{x}}$, the CPM differential detection method [14] is used to demodulate the symbol mapping bit $p_{2}$, and perform serial-to-parallel conversion processing to facilitate that combination of each group of bits in the next step, finally, combining each group of $p_{1}$ bits with $p_{2}$ to output serial data stream $A$-bits information.

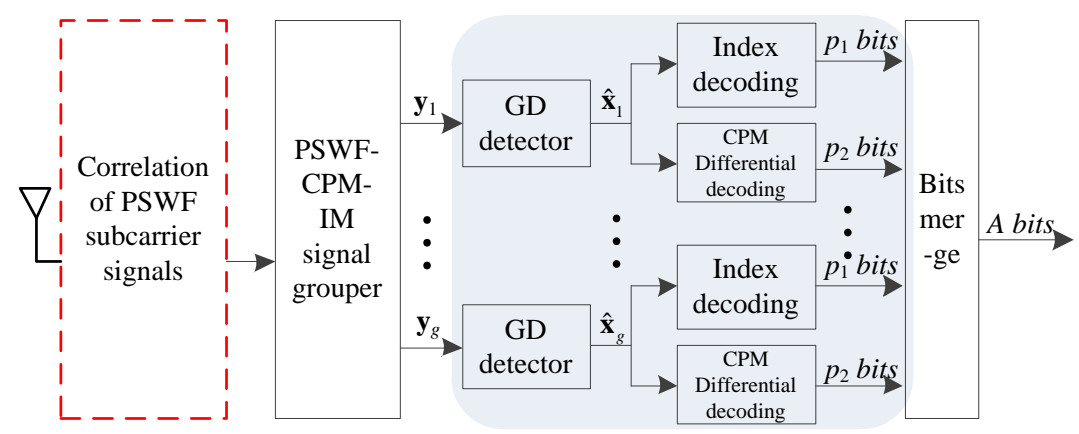

Fig. 3: Principle of PSWF-CPM-IM Signal Detection and Demodulation Method

\section{Performance Analysis}

The following is a simulation and analysis of the PSD, PAPR, BER performance and implementation complexity of the proposed PSWF-CPM-IM signal. In order to compare with OFDM signal under the same 
conditions, the influence of cyclic prefix on system performance is not considered here. For the convenience of illustration, the PSWF-CPM-IM signal is denoted as 'PSWF(CPM)-IM', and it is mainly compared with the modulation signal generated by pulse amplitude modulation (PAM) pre-modulation method used by PSWF signal in multi-carrier applications, represented as 'PSWF(PAM)-IM'.

\subsection{Power Spectral Density}

Parameter setting: in Figure 4. (a), the time bandwidth product $c$ of PSWF is $20 \mathrm{~Hz} \cdot \mathrm{s}, N=16, K=8$, oversampling points: $S=256$, binary number $W=2$. In Figure 4 . (b), $c=4 \mathrm{~Hz} \cdot \mathrm{s}, N=4, K=3, S=256, W=2$. The modulation index $h$ are $0.25,0.5,0.75$.

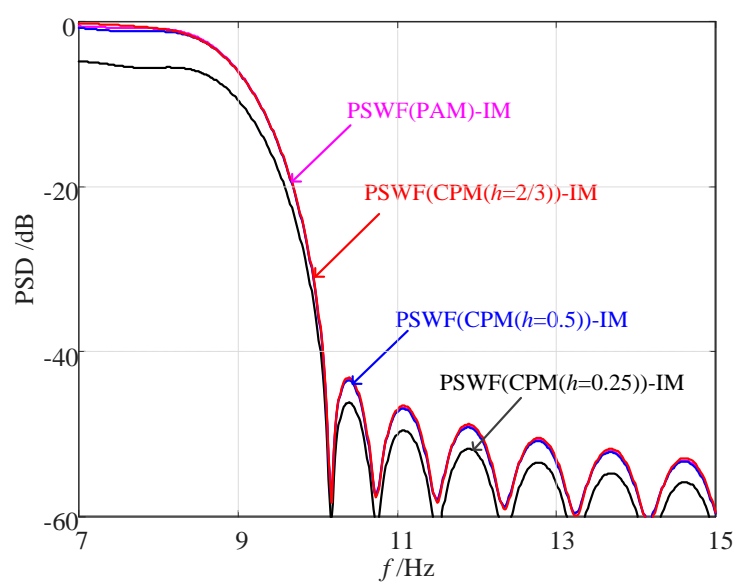

(a) PSWF(CPM)-IM and PSWF(PAM)-IM

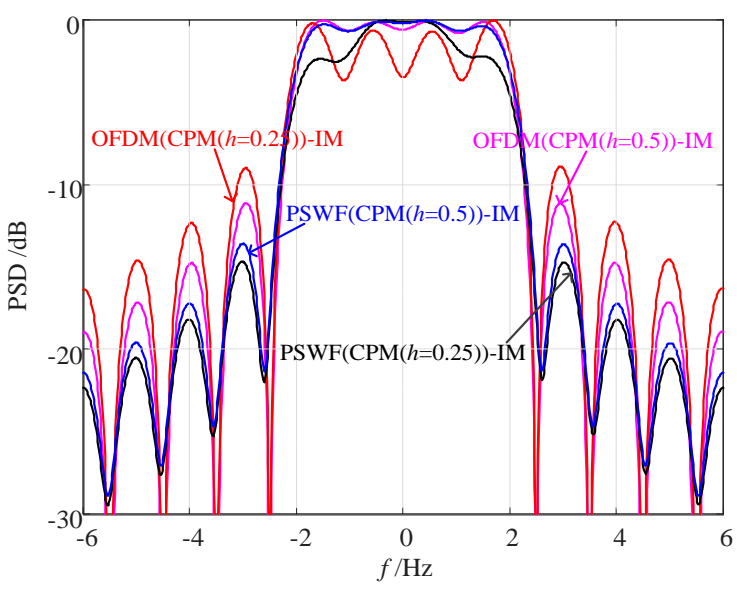

(b) PSWF(CPM)-IM and OFDM(CPM)-IM

Fig. 4: PSD of PSWF(CPM)-IM Modulated Signal

It can be seen from Figure 4. (a) that PSWF(CPM)-IM modulated signal have different PSD characteristics under different modulation index conditions, and will change with the change of CPM modulation index $h$, when $h=0.25$, the PSD of PSWF(CPM)-IM modulation signal decays quickly out of band, and when $h$ is greater than or equal to 0.5 ( $h$ is a constant exponent and $h \in(0,1)$ ), its power spectrum performance is basically equal. For PSWF(CPM)-IM modulation, when the constellation mapping method adopts CPM mapper, compared with the PSWF(PAM)-IM signal, the CPM mapper can change the out-ofband attenuation degree of the power spectral density of the modulated signal by adjusting its modulation index parameter $h$, and play a different degree of side-lobe suppression effect, and its main lobe energy concentration is almost unchanged.

\subsection{Peak-to-Average Ratio}

Parameter setting: $c=20 \mathrm{~Hz} \cdot \mathrm{s}, N=16, K=8, S=256, W=2$. The modulation index $h$ are $0.25,0.5,0.75$.

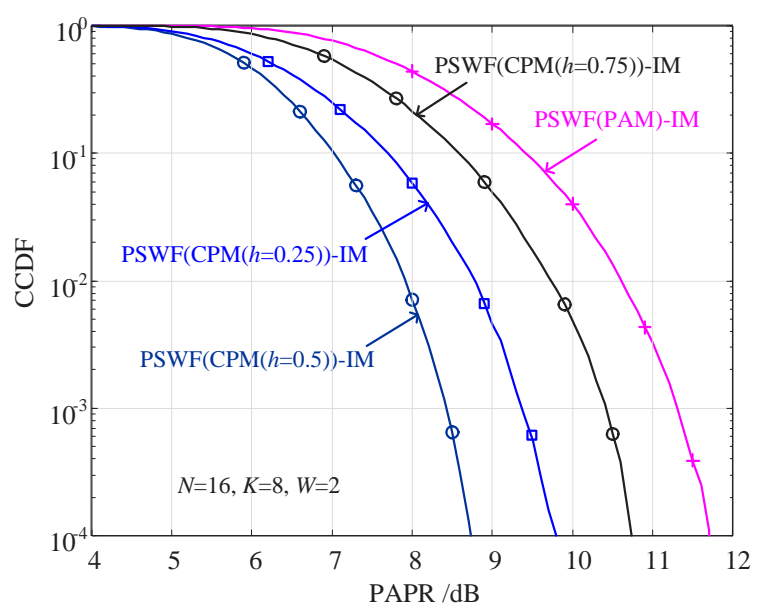

Fig. 5: PAPR of PSWF(CPM)-IM and PSWF(PAM)-IM Modulated Signal

It can be seen from Figure 5 that PSWF(CPM)-IM modulated signal mapped by CPM have different PAPR performances under different modulation indexes, especially, when the modulation index $h$ of CPM is 
0.5 and the complementary cumulative distribution function (CCDF) is $10^{-4}$, the PAPR value of PSWF(CPM)-IM modulation signal is about $8.70 \mathrm{~dB}$, which is about 3dB lower than that of PSWF(PAM)-IM modulation signal under the same parameters. See the Table 3 for PAPR values of PSWF(CPM)-IM modulated signals with different modulation indices when $\mathrm{CCDF}=10^{-4}$.

Table 3: PAPR value of PSWF(CPM)-IM modulation signal

\begin{tabular}{|c|c|c|c|c|}
\hline $\begin{array}{c}\text { Number of carriers } \\
(N=16, K=8)\end{array}$ & PSWF(PAM)-IM & $\begin{array}{c}\text { PSWF(CPM)-IM } \\
(h=0.5)\end{array}$ & $\begin{array}{c}\text { PSWF(CPM)-IM } \\
(h=0.25)\end{array}$ & $\begin{array}{c}\text { PSWF(CPM)-IM } \\
(h=0.75)\end{array}$ \\
\hline $\begin{array}{c}\text { CCDF }=10^{-4} \\
\text { PAPR values }\end{array}$ & $11.70 \mathrm{~dB}$ & $8.70 \mathrm{~dB}$ & $9.80 \mathrm{~dB}$ & $10.70 \mathrm{~dB}$ \\
\hline
\end{tabular}

\subsection{Performance of BER}

Comparing the BER performance of PSWF(CPM)-IM signal with PSWF(PAM)-IM signal when the modulation index $h$ of CPM is 0.5 , where $\varepsilon^{2}=0$ is perfect channel state information (CSI), $\varepsilon^{2}=0.01$ is fixed CSI, $E_{s} / N_{0}=10^{\left(E_{b} / N_{0}\right) / 10}$ and $\xi=1$ is the variable CSI.

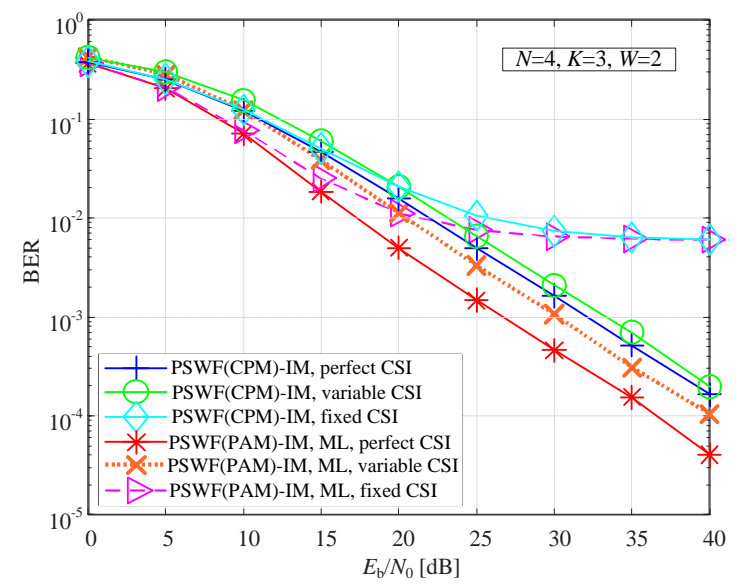

(a) BER of PSWF(CPM)-IM Signal $(N=4, K=3, W=2)$

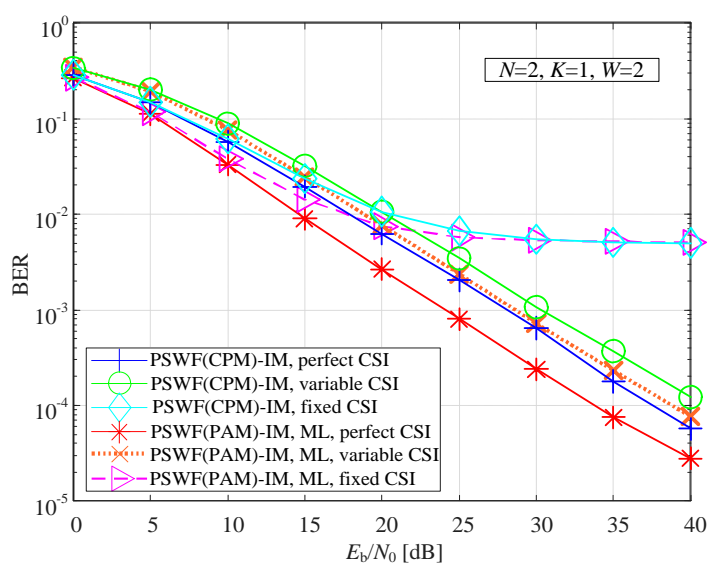

(b) BER of PSWF(CPM)-IM Signal ( $N=2, K=1, W=2)$

Fig. 6: BER of PSWF(CPM)-IM Signal Under Different CSI Conditions

From the comprehensive analysis of Figure 6, it can be seen that the signal detection performance is quite different from the perfect CSI by using ML detection algorithm, It can be seen from Figure 6. (b) that when $\mathrm{BER}=10^{-3}$, the difference between them is about $4.60 \mathrm{~dB}$, while the difference between them is about 2.10dB by GD detection method. This shows that compared with ML detection method, GD detection method makes PSWF(CPM)-IM signal insensitive to imperfect CSI and has certain stability, although the BER performance of PSWF(CPM)-IM signal decreases in an acceptable range. In addition, in the case of fixed CSI, the detection performance of GD gradually approaches the performance of ML detection algorithm with the increase of signal-to-noise ratio. Under variable CSI, the performance of GD detection is close to that of ML detection, and the difference is only about 1.20dB. With the increase of the number of $(N$, $K)$ combinations, the approach is more obvious, but the overall demodulation BER performance of PSWF(CPM)-IM system decreases slightly.

\subsection{Implementation Complexity}

In index modulation, the detector mainly completes the carrier position index and constellation symbol detection of grouped data, the ML detection algorithm has the best performance. According to literature [15], the complexity of addition and multiplication of ML is $O\left(2^{p 1} W^{K}\right)$, where $p_{1}$ is the number of index bits and $W$ is the modulation order. Its complexity increases exponentially with the increase of the number of index bits and $K$ (number of active sub-carriers), which is not easy to be realized in practical systems. If the absolute value is calculated once as two additions, the addition complexity of LLR detector is $O(W)$, and the multiplication complexity is $O(4 \mathrm{~W})$, which reduces the complexity of ML detection and achieves similar performance to ML detection, which is only proportional to the number of sub-carriers. The PSWF-CPM-IM signal detection end adopts GD detection method, which solves the index position bit $p_{1}$ through the maximum received energy of the corresponding $K$ active players, and its addition and multiplication 
complexity both are $O(W)$. Moreover, within the range of acceptable loss reliability, GD detection method has less sensitive to imperfect CSI.

\section{Conclusion}

This paper presents a multi-carrier index modulation method based on PSWF-CPM signal. According to CPM modulation index parameters, PSWF-CPM-IM signal can improve the PSD characteristics of existing modulated signals, by using CPM symbol mapping method, when PSWF sub-carriers $N$ is 16 , activation subcarriers $K$ is 8 and modulation index $h$ is 0.5, compared with PSWF(PAM)-IM signal, PSWF(CPM)-IM modulation signal can effectively reduce PAPR by about $3 \mathrm{~dB}$ under $\mathrm{CCDF}=10^{-4}$. At the receiving end, the GD algorithm based on energy detection combined with CPM differential symbol de-mapping can not only resist the sensitivity to the imperfect CSI a certain extent, but also has relatively low implementation complexity. However, with the increase of the number of PSWF sub-carriers, the BER performance decreases slightly compared with ML detection method. This method has certain reference value for promoting the better application of multi-carrier modulation technology based on PSWF signal in wireless communication field.

\section{References}

[1] E. Basar. Index modulation for 5G wireless networks. IEEE Communications Magazine. 2016, 54 (7): 168-175.

[2] M. Salah, O. A. Omer, U. S. Mohammed. Spectral efficiency enhancement based on sparsely indexed modulation for green radio communication. IEEE Access. 2019, 7 (3): 31913-31925.

[3] S. A. Nambi, and K. Giridar. Lower order modulation aided BER reduction in OFDM with index modulation. IEEE Communications Letters. 2018, 22 (8): 1596-1599.

[4] S. H. Alrubaee, M. Ismail, M. A. Altahrawi, et al. Filter bank multi-carrier modulation technique for vehicle-to vehicle communication. Journal of Communications. 2020, 15 (7): 566-571.

[5] F. P. Lu, H. X. Wang, C. H. Liu, et al. Strict parity symmetric prolate spheroidal wave functions signal construction and low complexity detection method. Sci. Sin. Inform. 2020, 50 (5): 766-776.

[6] T. Moumni. Solution of the energy concentration problem and application. Afrika Matematika. 2018, 29 (5): 929938.

[7] K. Mahata, and M. Hyder. Frequency estimation from arbitrary time samples. IEEE Transactions on Signal Processing. 2016, 64 (21): 5634-5643.

[8] M. D. Sanctis, E. Cianca, T. Rossi. Waveform design solutions for EHF broadband satellite communications. IEEE Communications Magazine. 2015, 53 (3): 18-23.

[9] J. G. Proakis, and M. Salehi. Digital communications (fifth edition). Publishing House of Electronics Industry, 2017.

[10] T. Aulin, and C. E. Sundberg. CPM-an efficient constant amplitude modulation scheme. International Journal of Satellite Communications. 1984, 2 (3): 161-186.

[11] E. Shafter, and K. R. Rao. CF technique with CPM mappers in OFDM system for reduction of PAPR. Proc. of IEEE Canadian Conference on Electrical and Computer Engineering. Vancouver, 2016, pp. 1-5.

[12] J. Crawford, and Y. Ko. Low complexity greedy detection method with generalized multicarrier index keying OFDM. Proc. of International Symposium on Personal, Indoor and Mobile Radio Communications. Hong Kong, 2015, pp. 688-693.

[13] T. V. Luong, and Y. Ko. A tight bound on BER of MCIK-OFDM with greedy detection and imperfect CSI. IEEE Communications Letter. 2017, 21 (12): 2594-2597.

[14] H. Harada, and R. Prasad. Simulation and software radio for mobile communications. Artech House, Inc., 2002.

[15] J. Zhang. Research on multicarrier systems with index modulation. Zhejiang University, 2017, pp. 11-19. 